\title{
Atrial fibrillation in cardiac resynchronization recipients with and without prior arrhythmic history. How much of arrhythmia is too much?
}

\author{
Radosław Lenarczyk, Ewa Jędrzejczyk-Patej, Mariola Szulik, Michał Mazurek, \\ Tomasz Podolecki, Jacek Kowalczyk, Oskar Kowalski, Beata Średniawa, \\ Zbigniew Kalarus; for the Triple-Site Versus Standard Cardiac \\ Resynchronization Trial (TRUST CRT) Investigators
}

Department of Cardiology, Congenital Heart Diseases and Electrotherapy, Silesian Medical University, Silesian Center for Heart Diseases, Zabrze, Poland

\begin{abstract}
Background: The aim of the study was to assess long-term incidence of atrial fibrillation $(A F)$ in cardiac resynchronization (CRT) recipients with and without prior arrhythmic history, factors predisposing to arrhythmia, as well as to evaluate the prognostic power of cumulative arrhythmia burden, duration of the longest episode and the number of episodes.

Methods: Device-collected data on AF episodes during 24 months in 96 participants of a randomized CRT-trial were analyzed (15\% in NYHA class $I V$, sinus rhythm, median left ventricular ejection fraction $24 \%$ and $Q R S 169 \mathrm{~ms}$ ). Blindly adjudicated major adverse cardiac events (MACE) and any-cause death were censoring variables.

Results: Two-year incidence of $A F$ was $70 \%$, including $66 \%$ of patients without previous $A F$ history. No baseline characteristics distinguished those who developed new onset AF. Percent of time spent in AF, but not number of episodes predicted mortality (adjusted hazard ratio [HR] $1.05 \pm 95 \%$ confidence interval CI 1.01-1.10) and MACE incidence (HR 1.03 \pm 1.01-1.07; $p=0.03$ ). Duration of the longest episode also predicted mortality (HR 1.06 $\pm 1.01-1.12$; both $p=0.03)$. Prognostic impact of AF load was marked only in patients with slower ventricular response (<98/min), but was independent from $C H A D S_{2}$ scores, pacing burden, or prior atrioventricular nodal ablation.

Conclusions: Seven out of 10 CRT-patients had AF within 2 years, including two-thirds of subjects without arrhythmic history. No baseline features distinguished those who developed new onset AF. Arrhythmia burden and duration of the longest episode, but not number of episodes influenced outcomes in CRT-patients, irrespectively from pacing burden or prior atrioventricular node ablation. (Cardiol J 2015; 22, 3: 267-275)
\end{abstract}

Key words: atrial fibrillation, cardiac resynchronization therapy, heart failure, prognosis, atrial fibrillation burden

\footnotetext{
Address for correspondence: Ewa Jędrzejczyk-Patej, MD, Department of Cardiology, Congenital Heart Diseases and Electrotherapy, Silesian Medical University, Silesian Center for Heart Diseases, ul. Skłodowskiej-Curie 9, 41-800 Zabrze, Poland, tel: +48 323733 682, fax: +48 323733 792; e-mail: ewajczyk@op.pl

Study data was presented at the Congress of the European Society of Cardiology, 30 August 2014-03 September 2014, Barcelona, Spain.
}

Received: 25.08.2014 Accepted: 11.11.2014 


\section{Introduction}

Atrial fibrillation (AF) carries an ominous prognosis in patients undergoing cardiac resynchronization therapy (CRT) [1-3]. Early studies used ambulatory electrocardiogram (ECG) or Holter recordings to detect AF [4]. Thus, many short, asymptomatic AF episodes may have been missed. Modern implantable devices are capable of detecting any, even short-lasting AF episodes and can store numerous arrhythmia-related parameters, like its cumulative load, number of episodes, or ventricular response. Although it has been suggested, that CRT can reduce AF incidence, data on its exact incidence, burden and predisposing factors are still scarce in patients undergoing biventricular pacing [5]. What is more, it is still a subject of controversy which parameters: cumulative time spent in arrhythmia, episode duration or the number of AF episodes have the prognostic significance.

The aim of our study was to assess a 2 -year arrhythmia incidence in CRT recipients with and without prior AF history and to identify factors predisposing to this arrhythmia. We aimed also at evaluating the prognostic power of cumulative $\mathrm{AF}$ burden, duration of the longest episode and the number of episodes.

\section{Methods}

\section{Patients}

Triple-Site Versus Standard Cardiac Resynchronization Therapy (TRUST CRT) was a single center, prospective, randomized trial to assess the effectiveness of triple-site pacing vs. standard cardiac resynchronization. The study protocol and procedural outcomes have been published previously $[6,7]$. The study included patients with symptomatic heart failure in New York Heart Association (NYHA) functional class III-IV despite optimal medical treatment, with $\mathrm{QRS}$ width $\geq 120 \mathrm{~ms}$, left ventricular ejection fraction (LVEF) $\leq 35 \%$ and significant $(\geq 40 \mathrm{~ms}$ ) inter- or intra-ventricular mechanical dyssynchrony. Patients had to be in sinus rhythm at enrollment, but a history of AF was not an exclusion criterion. Patients were randomized in a 1:1 ratio to conventional or triple-site resynchronization therapy with implantable cardioverter-defibrillator (ICD). The enrollment was accomplished in January 2010, and 100 consecutive patients were included.

Two patients were excluded up to 3 months after randomization due to lung cancer in one and alcohol abuse and non-compliance in the second, data of these patients were not further analyzed.
Two other patients in whom the implantation of resynchronization systems failed and who received ICD without device-based monitoring capabilities, were also excluded. Finally, data from 96 patients were used for the analysis.

The study protocol has been approved by a local Ethics Committee and complies with the Declaration of Helsinki. The trial has been registered on the Clinical Trials website (www.clinicaltrials.gov) and has been assigned with the clinical trial no NCT 00814840. Written informed consent was obtained from all study participants.

\section{Collection and analysis of clinical data}

Data on potential adverse events were collected during planned appointments ( 1 week, 1,3 , 6 months after implantation and every 6 months thereafter) and unscheduled ones throughout the whole observation period. They were retrieved from patients, relatives, witnesses, attending physicians, hospital records, outpatient notes, letters, death certificates, device memory, and all other available sources. The data obtained were subsequently classified by 2 independent experts, blinded to patients' treatment arm.

For the purpose of this analysis major adverse cardiovascular event (MACE) was considered a composite of hospitalization for heart failure (HF), heart transplantation, stroke, inadequate intervention of defibrillator, or any-cause death, whichever occurred first.

\section{Arrhythmic data}

Pacemakers' check-up was performed during every scheduled and unscheduled appointment. The whole memory content, including arrhythmic episodes, was downloaded on every visit (including post mortem interrogation, if possible). Files stored were subsequently converted into a format recognizable by commonly used database (Microsoft Excel, Microsoft, USA) and analyzed. Every, even the shortest AF episode was analyzed in this study, no duration cut-off was used to exclude short-lasting episodes.

Devices used in the trial (InSync Sentry 7298, Medtronic, Minneapolis, USA) detect AF using two main criteria: the activation patterns and timing and rate in both chambers (classified by an algorithm called PR Logic). In each ventricular cycle, the device incremented $\mathrm{AF}$ evidence count if all of the following parameters were identified: (1) atrioventricular pattern typical for a high atrial rate; (2) timing consistent with an atrial tachyarrhythmia (atrial activation sensed within $50 \mathrm{~ms}$ after or 
$80 \mathrm{~ms}$ prior to ventricular activation); (3) greater than 1:1 atrio-ventricular conduction.

Once the AF evidence count was greater than or equal to 6 , the arrhythmia was detected. For the purpose of this study, percent of cumulative time spent in $\mathrm{AF}$, duration of the longest episode, number of episodes, and mean ventricular rate during all episodes were calculated within 24 months after implantation. Further, these parameters were recalculated for the period between implantation and the first MACE event.

Appropriateness of every antiarrhythmic therapy delivered by defibrillator and stored in a device memory was assessed by two independent reviewers blinded to patients' treatment arm.

During the trial 17 patients died, in 4 of whom CRT-D interrogation was performed post-mortem. Median percent of the whole observation covered by interrogated devices memory was $100 \%$ (interquartile range $[\mathrm{IQR}]=0$ ).

\section{Statistical analysis}

Normality of distribution was tested with Kolgomorov-Smirnov test. The continuous parameters were presented as mean \pm standard deviation or median \pm IQR (depending on parameters' distribution), categorical variables as numbers and percentages. Comparison between the groups were performed with the $\chi^{2}$, t-Student or Mann-Whitney $\mathrm{U}$ tests, as appropriate.

Multivariate Cox regression models were constructed to assess independent predictive impact of time spent in arrhythmia, duration of the longest episode and number of episodes. Receiver-operating characteristics was used to calculate cut-off with the most balanced sensitivity and specificity of prognosticator. Generalized linear model for binomial data, with logit link function were used to test for interactions. All statistical analyses were performed using the Statistica software package (version 6.0, StatSoft Inc., Tulsa, OK, USA and version 10.0).

\section{Results}

\section{Study population}

The average age in the whole group was 61.5 years \pm IQR $13.5,22 \%$ of patients were female and $15 \%$ presented with NYHA class IV. Ischemic etiology was present in $60 \%$ of cases. The median LVEF was $24 \pm 4.5 \%$ and QRS duration was $169 \pm 30.5 \mathrm{~ms}$ before CRT-D implantation. At enrollment $13 \%$ of patients had previously diagnosed $\mathrm{AF}$, while $87 \%$ had no history of AF.

\section{Arrhythmia incidence in patients with and without arrhythmic history}

From among 13 patients with known AF before resynchronization, at least one episode of this arrhythmia occurred in $92 \%$ subjects within 2 years after implantation. In this group, the cumulative percent of time spent in arrhythmia was higher (median $0.21 \% \pm \mathrm{IQR} 7.7$ vs. $0.0001 \% \pm 0.05$ ), durations of the longest episodes were longer $(18.5 \pm 17.3 \mathrm{~h}$ vs. $0.02 \pm 1.9 \mathrm{~h}$; both $\mathrm{p}<0.001)$ and the number of AF episodes was greater $(23 \pm$ \pm 83 vs. $2 \pm 31, \mathrm{p}<0.05)$ than in patients without arrhythmic history (Fig. 1A-C). Adjusting for age, previously diagnosed AF posed a 2.75-time higher probability of experiencing this arrhythmia within further observation ( $p=0.002$; Fig. 1D).

On the other hand, from among 83 subjects in whom no AF was recognized prior to CRT, as many as $66 \%$ patients developed also this arrhythmia within the next 24 months. Of those, $36 \%$ had at least 1 episode lasting $>15 \mathrm{~min}$, in $20 \%$ the longest arrhythmic event lasted $>5.5 \mathrm{~h}$, and $13 \%$ had $>100$ episodes. In $3 \%$ of patients the arrhythmia progressed from paroxysmal to persistent form (vs. $15 \%$ of subjects with AF history; $p<0.05$ ). Assignment to the study group did not affect cumulative proportion of time in $\mathrm{AF}$ (median in the triple-site group $=0.0002$ vs. the conventional group $=$ $=0.008 \%$ ), duration of the longest episode ( $0.02 \mathrm{vs}$. $0.51 \mathrm{~h}$ ) or number of episodes ( $3 \mathrm{vs} .3$, all $\mathrm{p}=\mathrm{NS}$ ).

\section{Factors associated with propensity to $\mathrm{AF}$}

Patients with and without AF history had distinct baseline characteristics. Subjects with previously diagnosed arrhythmia had significantly (all $\mathrm{p}<0.05$ ) more frequently conduction block with non-left bundle branch block morphology in ECG (23\% vs. 6\%) and more compromised renal function (median serum creatinine $107 \mu \mathrm{mol} / \mathrm{L}$ vs. $91 \mu \mathrm{mol} / \mathrm{L}$ ) than patients without AF prior to CRT (Table 1). On the contrary, considering only subjects without AF history, no baseline features indicated those who developed eventually this arrhythmia within further follow-up. The only differences between those with $\mathrm{AF}$ and free of $\mathrm{AF}$ were, in fact, confined to more frequent use of loop diuretics (98\% vs. $85 \%$ ) and oral anticoagulants among patients with arrhythmia (Table 1).

\section{$\mathrm{AF}$ and outcome}

Considering the whole study population, patients who experienced at least one device-detected $\mathrm{AF}$ episode did not differ (all $\mathrm{p}=\mathrm{NS}$ ) from arrhythmia-free group with respect to 24-month mortality ( $18 \%$ vs. $17 \%$ ), rates of non-fatal $\mathrm{HF}$ 


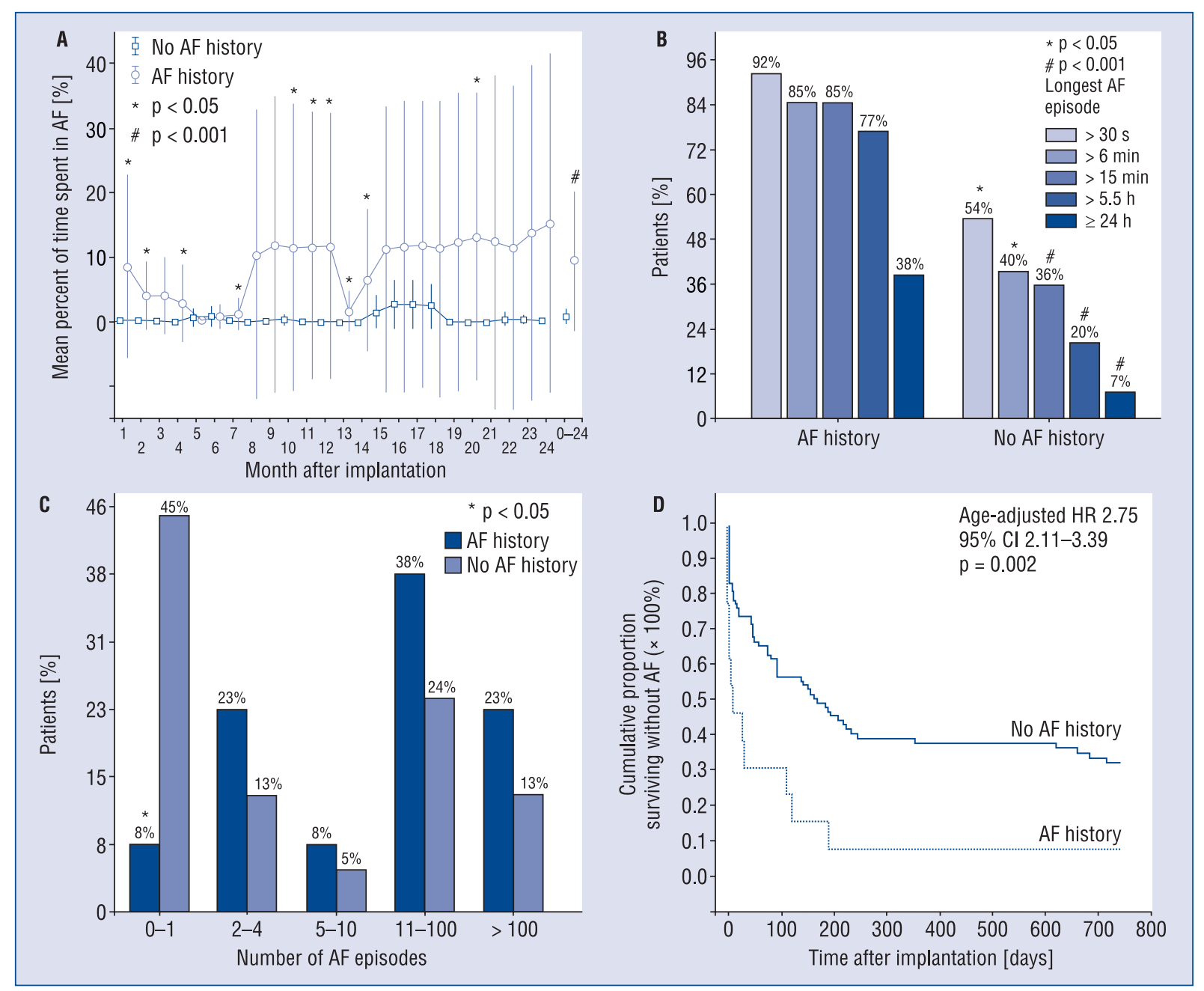

Figure 1. Atrial fibrillation (AF) incidence and burden in patients with and without arrhythmic history; A. Monthly and cumulative percent of time spent in AF. Boxes denote means, whiskers denote standard deviations; B. Duration of the longest arrhythmia episode; C. Number of arrhythmic episodes; D. Curves of cumulative survival without AF within 2 years after cardiac resynchronization (adjusted for age); $\mathrm{Cl}$ — confidence interval; $\mathrm{HR}$ - hazard ratio.

hospitalizations ( $31 \%$ vs. $17 \%)$, or strokes $(1.5 \%$ vs. $3 \%)$. However, the rates of inappropriate interventions of automatic defibrillator ( $61 \%$ vs. $27 \%$ ), as well as the incidence of combined MACE events $(69 \%$ vs. $41 \%$; both $\mathrm{p}<0.05)$ were both significantly higher in the arrhythmic group. Nine percent of AF patients underwent catheter ablation of atrioventricular junction within 24-month observation (vs. $0 \%$ in AF free group).

\section{Prognostic impact of cumulative time spent} in arrhythmia, longest episode duration and the number of episodes

Cumulative percent of time spent in $\mathrm{AF}$ and duration of the longest AF episode, but not the number of episodes were independent predictors of mortality (Table 2). Every additional percent of time spent in AF increased the risk of death by $5 \%$ (adjusted hazard ratio [HR] $1.05 \pm 95 \%$ confidence interval [CI] 1.01-1.10; $\mathrm{p}=0.03)$. In turn, every increase in episode duration by $1 \mathrm{~h}$ was associated with $6 \%$ increase in mortality risk (HR 1.06; 95\% CI 1.01-1.12; $\mathrm{p}=0.03$ ) (Table 2). Cumulative percent of time in arrhythmia, but not episode duration and number of episodes, was also independent predictor of MACE. The MACE-risk increased by $3 \%$ with any additional percent of time spent in AF (Table 3). One percent of time in $\mathrm{AF}$ had $35 \%$ sensitivity and $91 \%$ specificity in predicting future death. The optimally balanced cut-off for time in arrhythmia to predict mortality was $6.14 \%$ (sensitivity $29 \%$, specificity $97 \%$ ), and to predict MACE was $0.005 \%$ (sensitivity $60 \%$, specificity $76 \%$ ). 
Table 1. Baseline characteristics of groups with and without atrial fibrillation.

\begin{tabular}{|c|c|c|c|c|}
\hline & \multicolumn{2}{|c|}{ All patients $(n=96)$} & \multicolumn{2}{|c|}{ Without AF history $(n=83)$} \\
\hline & $\begin{array}{c}\text { AF history } \\
(n=13)\end{array}$ & $\begin{array}{c}\text { No AF history } \\
(n=83)\end{array}$ & $\begin{array}{l}\text { AF in FU } \\
(n=55)\end{array}$ & $\begin{array}{l}\text { No AF } \\
(n=28)\end{array}$ \\
\hline Age [year] & $62(16)$ & $61(13)$ & $61(15)$ & $62(13)$ \\
\hline Female & $2(15)$ & $19(23)$ & $12(22)$ & $7(25)$ \\
\hline NYHA class & $3(0)$ & $3(0)$ & $3(0)$ & $3(0)$ \\
\hline Ischemic etiology & $7(54)$ & $51(61)$ & $33(60)$ & $18(64)$ \\
\hline Arterial hypertension & $10(77)$ & $52(63)$ & $36(65)$ & $16(57)$ \\
\hline Diabetes mellitus & $4(31)$ & $30(36)$ & $21(38)$ & $9(32)$ \\
\hline QRS width [ms] & $167(26)$ & $170(33)$ & $172(31)$ & $162.5(43)$ \\
\hline LBBB & $10(77)$ & $78(94)^{*}$ & $52(94)$ & $26(93)$ \\
\hline $\mathrm{CHADS}_{2}$ score & $3(2)$ & $3(2)$ & $3(2)$ & $3(2)$ \\
\hline LVEF [\%] & $25(7)$ & $24(4)$ & $24(4)$ & $23.5(5)$ \\
\hline LVESV [mL] & $152(78)$ & $202(102)$ & $202(113)$ & $191.5(89)$ \\
\hline LVEDV [mL] & $212(87)$ & $268(120)$ & $268(135)$ & $260.5(113)$ \\
\hline Left atrium diameter [mm] & $44(10)$ & $44(10)$ & $46(11)$ & $41(8)$ \\
\hline Mitral incompetence grade & $1(2)$ & $2(2)$ & $2(2)$ & $2(2)$ \\
\hline Creatinine $[\mu \mathrm{mol} / \mathrm{L}]$ & $107(46)$ & $91(31)^{*}$ & $88(36)$ & $93.5(30)$ \\
\hline NT-proBNP [pg/mL] & $1,408.5(4,287)$ & $1,597(2,855)$ & $1,441(2,249)$ & $2,054(3,794)$ \\
\hline \multicolumn{5}{|l|}{ Medication at discharge: } \\
\hline Beta-blocker & $13(100)$ & $82(99)$ & $54(98)$ & $28(100)$ \\
\hline ACEI/ARB & $13(100)$ & $82(99)$ & $54(98)$ & $28(100)$ \\
\hline Aldosterone antagonist & $12(92)$ & $80(96)$ & $54(98)$ & $26(93)$ \\
\hline Loop diuretic & $11(85)$ & $78(94)$ & $54(98)$ & $24(86)^{+}$ \\
\hline Digoxin & $2(15)$ & $7(8)$ & $5(9)$ & $2(7)$ \\
\hline Amiodarone & $2(15)$ & $4(5)$ & $4(7)$ & 0 \\
\hline Oral anticoagulant & $11(85)$ & $9(11)^{*}$ & $8(14)$ & $1(4)$ \\
\hline Oral anticoagulants at 24 months $^{\#}$ & $9(90)$ & $24(35)^{*}$ & $23(50)$ & $1(4)^{+}$ \\
\hline
\end{tabular}

Continuous data are presented as median (interquartile range), dichotomic parameters as number (percent); ${ }^{*} \mathrm{p}<0.05 \mathrm{vs}$. group with $\mathrm{AF}$ history; ${ }^{+} p<0.05$ vs. group with $\mathrm{AF}$ during follow-up; \#79 patients survived the 24-month period: 10 with and 69 without $\mathrm{AF}$ history prior to cardiac resynchronization therapy. Among the latter group 46 developed arrhythmia within further follow-up, 23 remained AF-free; ACEI to cardiac resynchronization therapy. Among the latter group 46 developed arrhythmia within further follow-up, 23 remained AF-free; ACEI bundle branch block; LVEDV - left ventricular end-diastolic volume; LVEF — left ventricular ejection fraction; LVESV - left ventricular end-systolic volume; NT-proBNP - N-terminal propeptide of B-type natriuretic peptide; NYHA — New York Heart Association

\section{Factors modifying the prognostic role} of arrhythmia burden

Prognostic impact of percent of time spent in $\mathrm{AF}$ was influenced by mean ventricular rate during arrhythmia ( $\mathrm{p}$ for interaction $=0.002$; Pearson's $\chi^{2}$ for goodness of fit 64.9; degrees of freedom 65). In patients with low ventricular rate during arrhythmia $(\leq 98 / \mathrm{min})$ and higher than median percent of time $(>0.03 \%)$ spent in AF mortality was $18 \%$, while in those with lower than median arrhythmia burden death rate events were only $3 \%$. On the other hand, in subjects with high ventricular rate during $\mathrm{AF}$, mortality was less dependent on arrhythmia burden and significant (6\%) even in low arrhythmia burden (Fig. 2).
Opposite to ventricular rate, prognostic importance of arrhythmia load was independent from $\mathrm{CHADS}_{2}$ scores (p for interaction $=0.61$ ). Similarly, prognostic effect was independent neither from CRT burden $(\mathrm{p}=0.08)$, nor from previous ablation of atrioventricular node $(\mathrm{p}=0.06)$. Also, assignment to the treatment arm (triple-site vs. standard CRT) did not affect prognostic impact of time spent in $\mathrm{AF}$ ( $\mathrm{p}$ for interaction $=0.33$ ).

\section{Discussion}

Several studies analyzed device-stored data to assess the incidence of AF in CRT patients $[5,8-17]$. However, in the great majority of them 
Table 2. Multivariate Cox regression models for prediction of mortality.

\begin{tabular}{|c|c|c|}
\hline Variable & HR (95\% CI) & $\mathbf{P}$ \\
\hline \multicolumn{3}{|l|}{ Model 1} \\
\hline Age [year] & $0.97(0.91-1.03)$ & 0.36 \\
\hline Ischemic etiology & $1.91(0.58-6.32)$ & 0.29 \\
\hline LBBB & $1.20(0.14-10.14)$ & 0.86 \\
\hline LVEF [\%] ${ }^{*}$ & $1.11(0.96-1.27)$ & 0.15 \\
\hline Serum creatinine $(10 \mu \mathrm{mol} / \mathrm{L})^{*}$ & $0.96(0.84-1.10)$ & 0.60 \\
\hline NT-proBNP $(100 \mathrm{pg} / \mathrm{mL})^{*}$ & $1.01(1.0-1.02)$ & 0.01 \\
\hline QRS width $(10 \mathrm{~ms})^{*}$ & $0.83(0.66-1.05)$ & 0.12 \\
\hline Percent of time spent in AF [\%] & $1.05(1.01-1.10)$ & 0.03 \\
\hline \multicolumn{3}{|l|}{ Model 2} \\
\hline Age [year] & $0.99(0.93-1.05)$ & 0.72 \\
\hline Ischemic etiology & $1.63(0.48-5.48)$ & 0.43 \\
\hline LBBB & $1.12(0.13-9.64)$ & 0.92 \\
\hline LVEF [\%] ${ }^{*}$ & $1.07(0.93-1.22)$ & 0.34 \\
\hline Serum creatinine $(10 \mu \mathrm{mol} / \mathrm{L})^{*}$ & $0.98(0.86-1.12)$ & 0.78 \\
\hline NT-proBNP $(100 \mathrm{pg} / \mathrm{mL})^{*}$ & $1.01(1.0-1.02)$ & 0.01 \\
\hline QRS width $(10 \mathrm{~ms})^{*}$ & $0.80(0.63-1.03)$ & 0.08 \\
\hline Duration of the longest AF episode [h] & $1.06(1.01-1.12)$ & 0.03 \\
\hline \multicolumn{3}{|l|}{ Model 3} \\
\hline Age [year] & $0.97(0.91-1.04)$ & 0.40 \\
\hline Ischemic etiology & $1.76(0.52-5.97)$ & 0.36 \\
\hline LBBB & $1.71(0.20-14.45)$ & 0.62 \\
\hline LVEF $[\%]^{*}$ & $1.09(0.95-1.25)$ & 0.21 \\
\hline Serum creatinine $(10 \mu \mathrm{mol} / \mathrm{L})^{*}$ & $1.06(0.95-1.19)$ & 0.30 \\
\hline NT-proBNP $(100$ pg/mL)* & $1.01(1.00-1.02)$ & 0.01 \\
\hline QRS width $(10 \mathrm{~ms})^{*}$ & $0.87(0.70-1.09)$ & 0.23 \\
\hline Number of AF episodes [n] & $0.99(0.99-1.0)$ & 0.42 \\
\hline
\end{tabular}

*Baseline (preoperative) values; AF — atrial fibrillation; Cl — confidence interval; HR — hazard ratio; LBBB — left bundle branch block; LVEF — left ventricular ejection fraction; NT-proBNP - N-terminal propeptide of B-type natriuretic peptide; NYHA - New York Heart Association

monitoring periods were short, ranging from 3 to 13 months. To our knowledge, in only 2 studies device-collected arrhythmic data were gathered for over 2 years. In MADIT-CRT substudy, patients were monitored for the average of 2.9 years and $\mathrm{AF}$ incidence within this period ranged from $3 \%$ to $9 \%$ [8]. However, this trial included only patients with less advanced HF (NYHA class I/II). In the study by Borleffs et al. [9], 25\% out of 223 CRT recipients developed AF within 32 months. This study in turn excluded patients with previous AF history, who constitute the majority of subjects developing this arrhythmia within further observation [10].

Our data show that when monitoring CRT patients for 2 years and analyzing every AF episode, arrhythmia incidence among patients with previous arrhythmic history is $92 \%$. This indicates that almost every patient with AF-history still does have arrhythmia after CRT implantation. These findings stay in line with a previous report suggesting that resynchronization does not reduce $\mathrm{AF}$ incidence among patients with prior arrhythmia [10]. This finding can also potentially explain higher mortality risk among CRT recipients with AF history [3, 18]. Unexpectedly, at least one AF episode could also be found in as many as $66 \%$ of patients without previous arrhythmic history. To our knowledge, the incidence of $\mathrm{AF}$ in our study has been the greatest reported to date ( $70 \%$ of all study patients). Longer follow-up and inclusion of every, even the shortest $\mathrm{AF}$ episode ( $10 \%$ had the longest $\mathrm{AF}$ episode $\leq 30 \mathrm{~s}$ ), are the possible explanations for differences between our data and other reports. Virtually all previously published studies used some duration 
Table 3. Multivariate Cox regression models for prediction of major adverse cardiovascular events.

\begin{tabular}{|c|c|c|}
\hline Variable & $\operatorname{HR}(95 \% \mathrm{CI})$ & $\mathbf{P}$ \\
\hline \multicolumn{3}{|l|}{ Model 1} \\
\hline Age [year] & $0.99(0.96-1.03)$ & 0.88 \\
\hline Ischemic etiology & $0.57(0.32-1.0)$ & 0.05 \\
\hline LBBB & $0.68(0.27-1.72)$ & 0.41 \\
\hline LVEF [\%]* & $1.01(0.94-1.07)$ & 0.87 \\
\hline Serum creatinine $(10 \mu \mathrm{mol} / \mathrm{L})^{*}$ & $1.06(0.97-1.15)$ & 0.19 \\
\hline NT-proBNP $(100 \mathrm{pg} / \mathrm{mL})^{*}$ & $1.01(1.0-1.01)$ & 0.03 \\
\hline QRS width $(10 \mathrm{~ms})^{*}$ & $0.92(0.3-1.03)$ & 0.15 \\
\hline Percent of time spent in AF [\%] & $1.03(1.01-1.07)$ & 0.03 \\
\hline \multicolumn{3}{|l|}{ Model 2} \\
\hline Age [year] & $0.99(0.96-1.02)$ & 0.86 \\
\hline Ischemic etiology & $0.57(0.33-1.0)$ & 0.05 \\
\hline LBBB & $0.69(0.27-1.77)$ & 0.45 \\
\hline LVEF [\%]* & $0.99(0.93-1.06)$ & 0.86 \\
\hline Serum creatinine $(10 \mu \mathrm{mol} / \mathrm{L})^{*}$ & $1.05(0.97-1.14)$ & 0.22 \\
\hline NT-proBNP (100 pg/mL)* & $1.01(1.0-1.01)$ & 0.04 \\
\hline QRS width $(10 \mathrm{~ms})^{*}$ & $0.91(0.81-1.02)$ & 0.09 \\
\hline Duration of the longest AF episode [h] & $1.02(0.99-1.06)$ & 0.12 \\
\hline \multicolumn{3}{|l|}{ Model 3} \\
\hline Age [year] & $0.99(0.96-1.03)$ & 0.87 \\
\hline Ischemic etiology & $0.62(0.35-1.09)$ & 0.09 \\
\hline LBBB & $0.63(0.25-1.58)$ & 0.33 \\
\hline $\operatorname{LVEF}[\%]^{*}$ & $0.99(0.93-1.06)$ & 0.75 \\
\hline Serum creatinine $(10 \mu \mathrm{mol} / \mathrm{L}) *$ & $1.05(0.97-1.14)$ & 0.20 \\
\hline NT-proBNP $(100 \mathrm{pg} / \mathrm{mL})^{*}$ & $1.01(1.0-1.01)$ & 0.03 \\
\hline QRS width $(10 \mathrm{~ms})^{*}$ & $0.93(0.83-1.03)$ & 0.15 \\
\hline Number of AF episodes [n] & $1.0(0.99-1.01)$ & 0.34 \\
\hline
\end{tabular}

Abbreviations as in Table 2.

cut-offs to detect arrhythmia. However, the use of cut-points to diagnose AF can be confusing. Assuming that $>10 \mathrm{~min}$ burden/day is needed to detect arrhythmia, a hypothetical patient with daily AF load of 9 min would be classified by investigators as having no arrhythmia at all, while in reality, he would spent $0.6 \%$ of time (2.3 days every year) in $\mathrm{AF}$.

Some of the prior studies attempted to identify association between some specific level of $\mathrm{AF}$ burden and further prognosis in patients with pacemakers and ICD [19-21]. However, not only the analyzed indices differed between various studies (cumulative burden, specific duration of an episode), but also pre-specified cut-points were divergent. MOST investigators [19] found that device-detected episode of atrial high rate lasting $>5$ min predisposes to death, composite of death and stroke, and forecasts future $\mathrm{AF}$ in pacemaker patients. In ASSERT Trial [20], presence of AF episode of $>6$ min was associated with 2.5 -fold higher risk of stroke or systemic embolism in patients with pacemakers or ICD. TRENDS Trial [21] included subgroup of CRT patients, but no data were recalculated specifically for this subgroup. TRENDS investigators [21] found that in subjects with pacemaker, ICD or CRT who present with $\geq 1$ stroke risk factor, daily AF cumulative burden of $\geq 5.5 \mathrm{~h}$ within 30 days doubles the risk of stroke.

Contrary to the aforementioned trials, in our approach, arrhythmia load was treated as a continuous, rather than a dichotomized variable. Additionally, various arrhythmia characteristics were assessed to identify the most prognostically important ones. We found that irrespective of the number of episodes, only percent of time spent in 


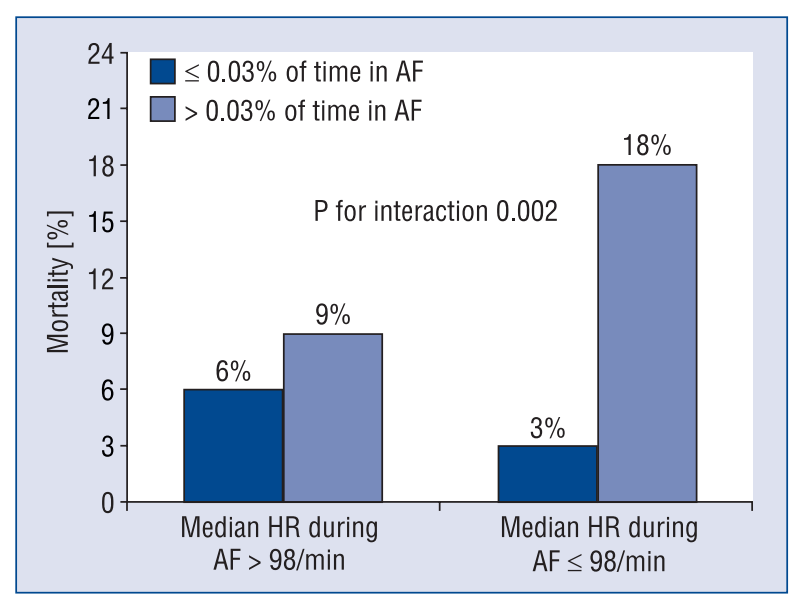

Figure 2. Predictive value of time spent in atrial fibrillation (AF) depending on heart rate (HR) during arrhythmia. Patients were dichotomized with respect to median values of two parameters: ventricular rate during $A F$ (median for whole study population $98 / \mathrm{min}$ ), and median cumulative percent of time spent in arrhythmia $(0.03 \%)$. Influence of arrhythmia burden was marked more in patients with slower heart rates.

AF had significant and independent prognostic role in CRT recipients. Duration of the longest episode predicted also the risk of death. Each additional percent in arrhythmia was moderately sensitive (35\%), but highly specific (91\%) in predicting mortality, increasing its risk by $5 \%$. Of note, as little as $0.005 \%$ of time spent in $\mathrm{AF}$ showed reasonable sensitivity and specificity in predicting MACE (mainly HF hospitalizations and defibrillator therapy). As opposed to duration of the longest episode or $\mathrm{cu}-$ mulative AF burden, percent of time in arrhythmia seems to be a more universal parameter, which may reflect better a time-varying risk factor associated with AF. AF-related risk does not probably remain stable, but increases with time in patients with progression to permanent $\mathrm{AF}$, and declines in subject free of AF for several months (what is mirrored by percent of time in arrhythmia). However, duration of the longest episode or cumulative AF burden can only increase (or, at best remain stable). What is more, cumulative arrhythmia burden and the longest episode duration are both hard to compare between patients with different observation times.

Our data indicate that prognostic impact of time in arrhythmia can be seen only in patients with lower ventricular rates, but is less important in the group of patients with uncontrolled rates during AF. This underscores previously reported importance of high ventricular rates during $\mathrm{AF}$, effect of which can overwhelm the prognostic im- pact of high arrhythmia burden [22]. Our results indicate that impact of various features of $\mathrm{AF}$ may vary in HF patients, depending on ventricular rate. In subjects in whom AF conducts with high ventricular rates, arrhythmia load is probably less important, as even short AF episodes with high ventricular rates can evoke $\mathrm{HF}$ decompensation or inappropriate defibrillator therapy. By contrast, patients with slow ventricular rates during $\mathrm{AF}$ usually remain hemodynamically stable during short episodes. In this group, the clinical manifestation of arrhythmia seems to be more time-dependent and sufficiently high AF load is probably needed before all the repercussions of irregular heart rate, loss of "atrial kick", thromboembolism and other AF consequences become apparent. However, these hypotheses need to be verified by further studies. On the other hand, time spent in AF exerted its prognostic effect independently from baseline $\mathrm{CHADS}_{2}$ scores. This finding is at odds with data by Botto et al. [23] who showed that arrhythmia burden acts as a prognosticator only in patients with intermediate (1-2 $\mathrm{CHADS}_{2}$ points), but not in high- or low-risk groups. Yet, our group included no patients with $\mathrm{CHADS}_{2} 0$ points (all of them had HF), and majority of them could be classified as belonging to the intermediate risk group. What is probably the most unexpected finding in our study is that the percent of time spent in AF acted unfavorably independently from CRT pacing burden and prior ablation of atrioventricular node. This result suggests that even in patients with high CRT pacing burden, or in those who underwent ablation of atrioventricular node, high percent of time spent in $\mathrm{AF}$ poses unfavorable prognosis. It corresponds with earlier data on independent detrimental role played by AF in CRT recipients and provokes to further attempt to verify the hypothesis, if aggressive rhythm control would be more beneficial than rate control in this group [24-28].

\section{Limitations of the study}

The study group was relatively small which could have led to underestimation of some important predictors. Resynchronization devices used in the trial did not store intracardiac electrograms during $\mathrm{AF}$, therefore it was impossible to verify the correctness of the devices' classification of detected episodes. In fact, some of the episodes could have been misdetected due to sensing problems, electrical noise, etc. However, despite of missed verification of appropriateness in MOST Trial, device-detected atrial high rate episodes carried unfavorable prognosis. 


\section{Conclusions}

As many as 7 out of 10 patients had AF within 2 years after CRT-D implantation, including twothirds of subjects without prior history of AF. No baseline features were helpful in predicting who would develop AF de novo. Cumulative percent of time spent in $\mathrm{AF}$ and duration of the longest episode, but not the number of AF episodes had significant and independent impact on further outcomes in CRT-patients. Prognostic impact of arrhythmia burden was marked only in patients with slow ventricular response, but was independent from $\mathrm{CHADS}_{2}$ scores, percent of biventricular pacing and prior nodal ablation.

Conflict of interest: Radosław Lenarczyk, MD, Oskar Kowalski, MD - received consultant fees from Medtronic and Biotronik, Mariola Szulik, MD - received lecturer fee from GE Healthcare, Beata Średniawa, MD - received consultant fees from Medtronic, other authors - no conflict of interests reported.

\section{References}

1. Molhoek SG, Bax JJ, Bleeker GB et al. Comparison of response to cardiac resynchronization therapy in patients with sinus rhythm versus chronic atrial fibrillation. Am J Cardiol, 2004; 94: 1506-1509.

2. Tolosana JM, Hernandez Madrid A, Brugada J et al. SPARE Investigators. Comparison of benefits and mortality in cardiac resynchronization therapy in patients with atrial fibrillation versus patients in sinus rhythm (Results of the Spanish Atrial Fibrillation and Resynchronization [SPARE] Study). Am J Cardiol, 2008; 102: 444-449.

3. Bai R, Di Biase L, Elayi C et al. Mortality of heart failure patients after cardiac resynchronization therapy: Identification of predictors. J Cardiovasc Electrophysiol, 2008; 1912: 1259-1265.

4. Hoppe UC, Casares JM, Eiskjaer $\mathrm{H}$ et al. Effect of cardiac resynchronization on the incidence of atrial fibrillation in patients with severe heart failure. Circulation, 2006; 114: 18-25.

5. Hügl B, Bruns HJ, Unterberg-Buchwald $\mathrm{C}$ et al. Atrial fibrillation burden during the post-implant period after CRT using device-based diagnostics. J Cardiovasc Electrophysiol, 2006; 17: 813-817.

6. Lenarczyk R, Kowalski O, Sredniawa B et al. Triple-site versus standard cardiac resynchronization therapy study (TRUST CRT): Clinical rationale, design, and implementation. J Cardiovasc Electrophysiol, 2009; 20: 658-662.

7. Lenarczyk R, Kowalski O, Sredniawa B et al. Implantation feasibility, procedure-related adverse events and lead performance during 1-year follow-up in patients undergoing triple-site cardiac resynchronization therapy: A substudy of TRUST CRT randomized trial. J Cardiovasc Electrophysiol, 2012; 23: 1228-1236.

8. Brenyo A, Link MS, Barsheshet A et al. Cardiac resynchronization therapy reduces left atrial volume and the risk of atrial tachyarrhythmias in MADIT-CRT (Multicenter Automatic Defibrillator Implantation Trial with Cardiac Resynchronization Therapy). J Am Coll Cardiol, 2011; 58: 1682-1689.

9. Borleffs CJ, Ypenburg C, van Bommel RJ et al. Clinical importance of new-onset atrial fibrillation after cardiac resynchronization therapy. Heart Rhythm, 2009; 6: 305-310.

10. Adelstein EC, Saba S. Burden of atrial fibrillation after cardiac resynchronization therapy. Am J Cardiol, 2007; 100: 268-272.
11. Saxon LA, Greenfield RA, Crandall BG, Nydegger CC, Orlov M, VAN Genderen R. Results of the multicenter RENEWAL 3 AVT clinical study of cardiac resynchronization defibrillator therapy in patients with paroxysmal atrial fibrillation. J Cardiovasc Electrophysiol, 2006; 17: 520-525.

12. Lellouche N, De Diego C, Vaseghi M et al. Cardiac resynchronization therapy response is associated with shorter duration of atrial fibrillation. Pacing Clin Electrophysiol, 2007; 30: 1363-1368.

13. Puglisi A, Gasparini M, Lunati M et al. Persistent atrial fibrillation worsens heart rate variability, activity and heart rate, as shown by a continuous monitoring by implantable biventricular pacemakers in heart failure patients. J Cardiovasc Electrophysiol, 2008; 19: 693-701.

14. Leclercq C, Padeletti L, Cihák R et al. Incidence of paroxysmal atrial tachycardias in patients treated with cardiac resynchronization therapy and continuously monitored by device diagnostics. Europace, 2010; 12: 71-77.

15. Caldwell JC, Contractor H, Petkar S et al. Atrial fibrillation is under-recognized in chronic heart failure: Insights from a heart failure cohort treated with cardiac resynchronization therapy. Europace, 2009; 11: 1295-1300.

16. Santini M, Gasparini M, Landolina M et al. Device-detected atrial tachyarrhythmias predict adverse outcome in real-world patients with implantable biventricular defibrillators. J Am Coll Cardiol, 2011; 57: 167-172.

17. Shanmugam N, Boerdlein A, Proff J et al. Detection of atrial highrate events by continuous home monitoring: Clinical significance in the heart failure-cardiac resynchronization therapy population. Europace, 2012; 14: 230-237.

18. Boven NV, Theuns D, Bogaard K et al. Atrial fibrillation in cardiac resynchronization therapy with a defibrillator: A risk factor for mortality, appropriate and inappropriate shocks. J Cardiovasc Electrophysiol, 2013; 24: 1116-1122.

19. Glotzer TV, Hellkamp AS, Zimmerman J et al. Atrial high rate episodes detected by pacemaker diagnostics predict death and stroke: Report of the Atrial Diagnostics Ancillary Study of the MOde Selection Trial (MOST). Circulation, 2003; 107: 1614-1619.

20. Healey JS, Connolly SJ, Gold MR et al. Subclinical atrial fibrillation and the risk of stroke. N Engl J Med, 2012; 366: 120-129.

21. Glotzer TV, Daoud EG, Wyse DG et al. The relationship between daily atrial tachyarrhythmia burden from implantable device diagnostics and stroke risk: The TRENDS study. Circ Arrhythm Electrophysiol, 2009; 2: 474-480.

22. Boriani $\mathrm{G}$, Gasparini $\mathrm{M}$, Landolina $\mathrm{M}$ et al. Incidence and clinical relevance of uncontrolled ventricular rate during atrial fibrillation in heart failure patients treated with cardiac resynchronization therapy. Eur J Heart Fail, 2011; 13: 868-876.

23. Botto GL, Padeletti L, Santini M et al. Presence and duration of atrial fibrillation detected by continuous monitoring: Crucial implications for the risk of thromboembolic events. J Cardiovasc Electrophysiol, 2009; 20: 241-248.

24. Jędrzejczyk-Patej E, Lenarczyk R, Pruszkowska P et al. Long-term outcomes of cardiac resynchronization therapy are worse in patients who require atrioventricular junction ablation for atrial fibrillation than in those with sinus rhythm. Cardiol J, 2014; 21: 309-315.

25. Tolosana JM, Arnau AM, Madrid AH et al. Cardiac resynchronization therapy in patients with permanent atrial fibrillation. Is it mandatory to ablate the atrioventricular junction to obtain a good response? Eur J Heart Fail, 2012; 14: 635-641.

26. Tolosana JM, Trucco E, Khatib M et al. Complete atrioventricular block does not reduce long-term mortality in patients with permanent atrial fibrillation treated with cardiac resynchronization therapy. Eur J Heart Fail, 2013; 15: 1412-1418.

27. Hsu LF, Jaïs P, Sanders P et al. Catheter ablation for atrial fibrillation in congestive heart failure. N Engl J Med, 2004; 351: 2373-2383.

28. Khan MN, Jaïs P, Cummings J et al. Pulmonary-vein isolation for atrial fibrillation in patients with heart failure. N Engl J Med, 2008; 359: 1778-1785. 